\title{
A Theory of Acoustics in Solar Energy
}

\section{Himanshu Dehra}

House \# 596, Sector 16-A, Faridabad, Haryana, India.

Email: anshu_dehra@hotmail.com

Received January $12^{\text {th }}, 2013$; revised February $13^{\text {th }}, 2013$; accepted February $23^{\text {th }}, 2013$

\begin{abstract}
A novel theory of acoustics in solar energy supporting the principle of source and sink of solar energy is presented. The significance of the theory is in ascertaining the aftermaths of turning off solar energy. An amplifier constituting of a parallel plate photovoltaic device connected to a potentiometer is illustrated. It was constructed with a pair of glass coated photovoltaic modules and polystyrene filled plywood board as back panel with air ventilation through a parallel plate channel of amplifier. The sample results obtained from experiments and simulation model are presented to support hypothesis of acoustics for a parallel plate photovoltaic device connected to a potentiometer. A phenomenon of photovoltaic amplification is formulated.
\end{abstract}

Keywords: Photoelectric Effect; Loudspeaker; Plasma Torch; Photovoltaic Amplification; Solar Energy

\section{Source and Sink of Solar Energy}

The solar energy is radiated from the sun and the earthatmosphere system absorbs a portion of its incident energy. In addition to the energy received from the sun, the surface of the earth is warmed by a heat flux from its interior that results, primarily, from the decay of radioactive isotopes. The tides, which are a consequence of the earth-moon system, result in viscous friction, another energy input that affects the surface energy balance. To the extent that the earth is not changing, the heat radiated by the surface of the earth is equal to the sum of the heat inputs.

The solar energy is absorbed by planet earth and its surrounding environment viz., earth surface, earth atmosphere, forests, farms, rivers, ponds, lakes \& seas, living beings and civil structures (e.g. buildings, green houses, thermal power plants, collectors, panels, roads, bridges, ports, canals) [1]. Life on earth is completely dependent upon the energy radiated by the sun. All biological processes are either directly or indirectly dependent upon photosynthesis. By photosynthesis green plants convert solar energy into chemical energy. The solar radiation sources are classified as per type of wave of interference [1]. The absorbed sources of waves of interference of particle surface are light, sound, heat, electricity, fluid and fire, wich are function of absorptivity for a solar intensity.

The earth's land surface, oceans, and atmosphere absorb solar radiation and this raises their temperature. The earth and its earth-moon system is the sink of the solar energy. Incident solar energy is the dominant energy input. The radiation absorbed by the earth depends upon the earth's cross-sectional area perpendicular to the sun's flux. The earth is behaving as a black body radiator. The radiative temperature of the earth-atmosphere system determines the actual power radiated by the earth.

\section{Solar Energy Acoustics}

The amplitude of a solar energy wave is defined as the power storage per unit area per unit time. The solar power is stored in a packet of solar energy wave of unit cross sectional area and of length $s$, the speed of light. Therefore, the solar power intensity $I$ is the product of total power storage capacity for a packet of solar energy wave and the speed of light. The logarithm of two solar power intensities, $I_{1}$ and $I_{2}$, gives power difference for two solar power intensities. It is mathematically expressed as [2]:

$$
\text { Sol }=\log \left(I_{1}\right)\left(I_{2}\right)^{-1}
$$

where, Sol is a dimensionless logarithmic solar noise unit. Mathematically, decisol (dS) is more convenient for solar power systems. Since a decisol (dS) is $1 / 11$ th unit of a Sol, it is mathematically expressed by the equation:

$$
\mathrm{dS}=11 \log \left(I_{1}\right)\left(I_{2}\right)^{-1}
$$

The above concepts of acoustics in solar energy are similar to the theory of sound [3]. This anticipated theory of acoustics in solar energy is mathematically extensible for determining various acoustical expressions which has been supported by experimental and modeling results for a parallel plate photovoltaic device connected to a potentiometer. 


\section{Experiments and Simulation}

The full scale experimental setup for a parallel plate photovoltaic device connected to a potentiometer was installed in an outdoor room facility [4]. The schematic of the experimental setup is illustrated in Figure 1. An amplifier was built with a pair of glass coated photovoltaic (PV) modules forming a parallel plate duct with a plywood board and connected to a potentiometer. A potentiometer, a wire-wound variable resistor of up to $50 \Omega$ was a wire-wound circular coil with a sliding knob contact. It was used to vary electrical resistance across connected PV modules without interrupting the current. The characteristics of a parallel plate photovoltaic device connected to a potentiometer were established by varying electrical resistance with rotation of knob of a potentiometer. The current-voltage measurements were obtained for determining electric power output with a series electrical circuit connection of a pair of vertically inclined PV modules installed on a wooden frame.

The temperatures were measured as a function of volume of a parallel plate photovoltaic device. The nonlinear thermal results include measurements of temperatures for PV modules, insulating panel and ventilated air column in the wooden frame. The air velocities were developed in the ventilated air column for transportation of heat both as a measure of buoyancy and fan induced hybrid ventilation. The thermal measurement results of temperatures of various components of PV device, ambient air and room air temperatures, air velocities and solar intensities are presented in Tables $\mathbf{1}$ and $\mathbf{2}$.

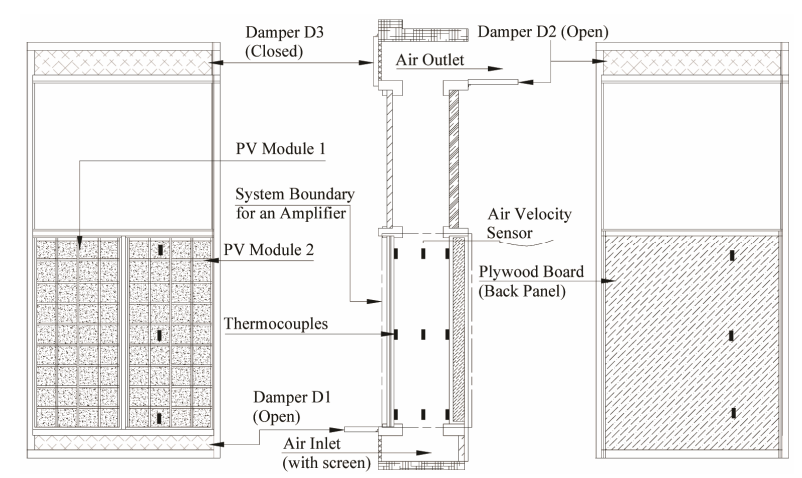

(a)

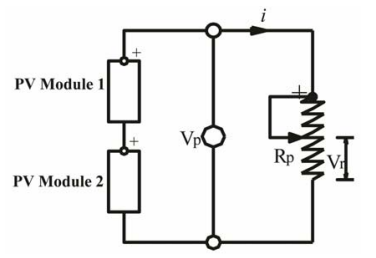

(b)

Figure 1. Schematic of experimental setup for a parallel plate photovoltaic device connected to a potentiometer: (a) location of sensors; (b) electrical circuit diagram.
The results of the power output from a potentiometer with rotation of circular knob are illustrated in Figure 2.

A simulation model for prediction of temperature distributions varying with volume of a parallel plate photovoltaic device was developed. The model has been used to predict temperature distributions at pre-defined locations in PV module, plywood board and air flowing through a parallel plate channel. The results of the temperature plots are illustrated from Figure 3.

\section{Photovoltaic Amplification}

The phenomenon of photovoltaic amplification is observed from the graphs of Figures 2 and 3. The gain in steady state electrical and thermal functions for a photovoltaic device is a factor of its volume or resistance. This operational characteristic is similar to operation of a loudspeaker. The electrical analog has been used to describe the resonance phenomenon for equivalent mechanical, hydraulic and thermal systems of parallel plate photovoltaic device connected to a potentiometer.

\subsection{Series Resonance}

For the case of series resonance, the equilibrium frequency $w_{0}$ is written as [5]:

$$
w_{0}=1 / \sqrt{L C}
$$

The frequency $w_{0}$ is called the resonant frequency. Its value is a function of two energy storing elements of the

Table 1. Input values.

\begin{tabular}{cccccc}
\hline Run No. & $\mathrm{S}\left(\mathrm{W} \cdot \mathrm{m}^{-2}\right)$ & $\mathrm{E}_{\mathrm{p}}(\mathrm{W})$ & $\mathrm{T}_{\mathrm{o}}\left({ }^{\circ} \mathrm{C}\right)$ & $\mathrm{T}_{\mathrm{s}}\left({ }^{\circ} \mathrm{C}\right)$ & $\mathrm{v}\left(\mathrm{m} \cdot \mathrm{s}^{-1}\right)$ \\
\hline 1 & 697.5 & 31.0 & 13.5 & 22.1 & 0.451 \\
2 & 725.4 & 31.1 & 15.9 & 22.9 & 0.362 \\
\hline
\end{tabular}

Table 2. Thermal measurement results.

\begin{tabular}{cccccccccc}
\hline $\begin{array}{c}\text { Run } \\
\text { No. }\end{array}$ & $\begin{array}{c}\mathrm{T}_{\mathrm{p}}(\mathrm{b}) \\
\left({ }^{\circ} \mathrm{C}\right)\end{array}$ & $\begin{array}{c}\mathrm{T}_{\mathrm{p}}(\mathrm{m}) \\
\left({ }^{\circ} \mathrm{C}\right)\end{array}$ & $\begin{array}{c}\mathrm{T}_{\mathrm{p}}(\mathrm{t}) \\
\left({ }^{\circ} \mathrm{C}\right)\end{array}$ & $\begin{array}{c}\mathrm{T}_{\mathrm{b}}(\mathrm{b}) \\
\left({ }^{\circ} \mathrm{C}\right)\end{array}$ & $\begin{array}{c}\mathrm{T}_{\mathrm{b}}(\mathrm{m}) \\
\left({ }^{\circ} \mathrm{C}\right)\end{array}$ & $\begin{array}{c}\mathrm{T}_{\mathrm{b}}(\mathrm{t}) \\
\left({ }^{\circ} \mathrm{C}\right)\end{array}$ & $\begin{array}{c}\mathrm{T}_{\mathrm{a}}(\mathrm{b}) \\
\left({ }^{\circ} \mathrm{C}\right)\end{array}$ & $\begin{array}{c}\mathrm{T}_{\mathrm{a}}(\mathrm{m}) \\
\left({ }^{\circ} \mathrm{C}\right)\end{array}$ & $\begin{array}{c}\mathrm{T}_{\mathrm{a}}(\mathrm{t}) \\
\left({ }^{\circ} \mathrm{C}\right)\end{array}$ \\
\hline 1 & 34.5 & 33.01 & 36.2 & 20.2 & 24.4 & 27.6 & 18.7 & 19.3 & 22.5 \\
2 & 32.5 & 33.3 & 35.7 & 20.2 & 23.9 & 29.1 & 18.3 & 19.7 & 23.3 \\
\hline
\end{tabular}

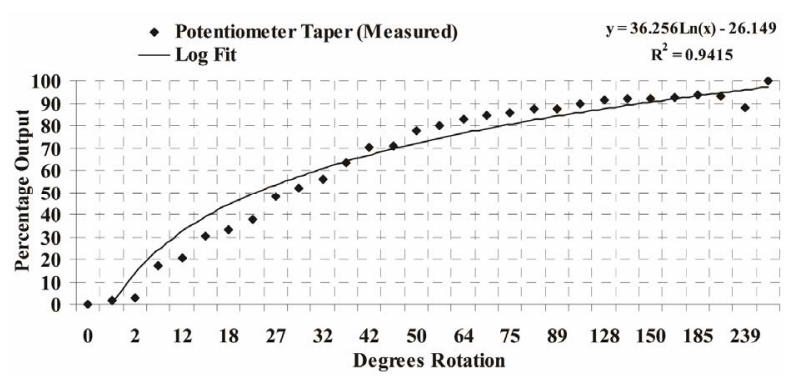

Figure 2. Potentiometer Taper (Measured) with percentage voltage output. 

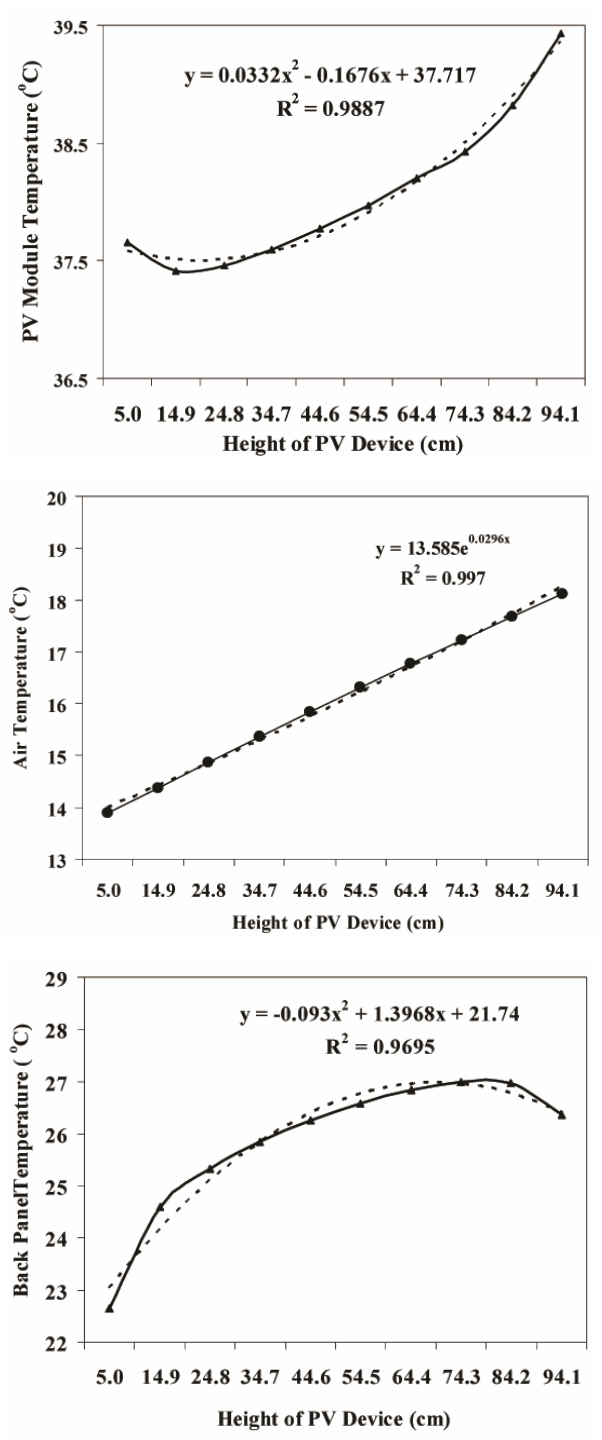

Figure 3. Temperature plots with height.

system. At resonance the impedance of the circuit is a minimum, and is equal to R. Consequently, when a series $\mathrm{L}-\mathrm{C}-\mathrm{R}$ circuit is at resonance, the current is a maximum and is also in time phase with the voltage. The power factor is unity. For operation at a frequency below $w_{0}$ the resultant imaginary part of impedance is capacitive so that the current leads the voltage. When $w>w_{0}$, the inductive reactance prevails, so that the current then lags the voltage. When the ratio of inductive reactance to resistance in a series L-C-R circuit is high, a very rapid rise of current to the maximum value occurs in the vicinity of the resonant frequency.

\subsection{Band Width}

The procedure as described in is used herewith for determining bandwidth at resonance. The bandwidth is defined as the range of frequency for which the power de- livered to $R$ is greater than or equal to $P_{R} / 2$, where $P_{R}$ is the power delivered to $R$ at resonance. As the magnitude of the current at each half-power point during resonance is same, we can write:

$$
i_{1}^{2} R=\frac{1}{2} i_{0}^{2} R=\frac{1}{2} i_{2}^{2} R
$$

where, subscript 1 denotes the lower half-power point and subscript 2 the higher half-power point. The expressions for $i_{1}$ and $i_{2}$ are then written as:

$$
i_{1}=i_{2}=i_{0} / \sqrt{2}=0.707 i_{0}
$$

The bandwidth is defined during resonance as that range of frequency over which the magnitude of the current is equal to or greater than 0.707 of the current at resonance. For $w_{0} L / R=1 / 2$, the frequency at the lower half-power point is denoted by $w_{1}$ and that of the upper half power point is denoted by $w_{2}$. Hence the bandwidth is $w_{2}-w_{1}$. Since the current at the half-power points is io $/ \sqrt{2}$, it follows that the magnitude of the impedance must be equal to $\sqrt{2} R$ to yield this current. The reactance during resonance is at the lower half-power frequency $X_{1}$, is then written as:

$$
X_{1}=w_{1} L-1 / W_{1} C=-R
$$

The minus sign appears on the right side of the equation because below resonance the capacitive reactance exceeds the inductive reactance. Rearranging Equation (6), leads to:

$$
w_{1}^{2}+(R / L) w_{1}-1 / L C=0
$$

The expressions for positive upper half-power and lower half-power frequency are written as:

$$
\begin{aligned}
& w_{1}=-(R / 2 L)+\sqrt{(R / 2 L)^{2}+(1 / L C)} \\
& w_{2}=-(R / 2 L)+\sqrt{(R / 2 L)^{2}+(1 / L C)}
\end{aligned}
$$

Therefore the expression for the bandwidth becomes, from Equations (8) and (9),

$$
w_{b w}=w_{2}-w_{1}=R / L
$$

\subsection{Quality Factor $Q_{0}$ of the RLC Circuit}

By forming the ratio of the resonant frequency to the bandwidth, a quality factor to measure the selectivity or sharpness of tuning of the series RLC circuit is obtained:

$$
Q_{0}=\left(w_{0} / w_{b w}\right)
$$

When Equation (10) is inserted into Equation (11):

$$
Q_{0}=w_{0} L / R=X_{L 0} / R
$$

By making use of the knowledge that $Q_{0}$ is very large in many resonant circuits and systems, the lower and upper half-power points are expressed in terms of the 
resonant frequency and the bandwidth.

The Equation (8) is re-written as:

$$
w_{1}=-(R / 2 L)+\sqrt{(R / 2 L)^{2}+\left(Q_{0}^{2} R^{2} / L^{2}\right)}
$$

For values of $Q_{0}$ which are 5 or greater very little error is made by writing:

$$
w_{1}=-(R / 2 L)+(R / L) Q_{0}
$$

Inserting Equation (10) into Equation (14) yields:

$$
w_{1}=w_{0}-\frac{1}{2} w_{b w}
$$

Similarly, expression for $w_{2}$ is written as:

$$
w_{2}=w_{0}+\frac{1}{2} w_{b w}
$$

The definition of quality factor is also expressed in terms of power storage. The instantaneous expression for the forced solution of current at resonance is:

$$
i_{0}=i_{m 0} \cos (w t)
$$

The total power stored by the circuit in both $L$ and $C$ is expressed as:

$$
W=\frac{1}{2} L i_{0}^{2}+\frac{1}{2} C V_{c 0}^{2}
$$

Inserting the appropriate expressions for $i_{0}$ and $V_{c 0}$ results in:

$$
\begin{gathered}
W=\frac{1}{2} L i_{m 0}^{2} \cos ^{2}\left(w_{0} t\right)+\frac{1}{2} L i_{m 0}^{2} \sin ^{2}\left(w_{0} t\right) \\
W=\frac{1}{2} L i_{m 0}^{2}=L i_{0}^{2}
\end{gathered}
$$

where, $i_{m 0}$ is maxima and $i_{0}$ is the rms value of current at resonance. The energy stored at resonance is a constant quantity. From Equation (12), the expression for $Q_{0}$ is written as [3]:

$$
Q_{0}=\left(w_{0} L i_{0}^{2} / R i_{0}^{2}\right)=\left(2 \pi f L i_{0}^{2} / R i_{0}^{2}\right)=2 \pi i_{0}^{2} L\left(i_{0}^{2} R / f_{0}\right)^{-1}
$$

It is written in terms of stored energy and dissipated energy as:

$$
Q_{O}=2 \pi\left(\frac{\text { total energy stored }}{\text { energy dissipated per cycle }}\right)
$$

It is applicable to any resonant system regardless of its composition.

\subsection{Band Width for Parallel Resonance}

For the parallel case, the equation for lower half power frequency $w_{1}$ is written as:

$$
w_{1} C-1 / w_{1} L=-1 / R
$$

The quadratic equation is written as:

$$
w_{1}^{2}+(1 / R C) w_{1}-1 / L C=0
$$

Ignoring the negative solution, the desired solution is:

$$
w_{1}=-1 / 2 R C+\sqrt{(1 / 2 R C)^{2}+1 / L C}
$$

The expression for the upper half-power frequency is:

$$
w_{2}=1 / 2 R C+\sqrt{(1 / 2 R C)^{2}+1 / L C}
$$

The expression for the bandwidth is:

$$
w_{b w}=w_{2}-w_{1}=1 / R C
$$

The expression for quality factor is written as:

$$
Q_{0}=w_{0} / w_{b w}=w_{0} R C
$$

\section{Discussions}

The incident short wavelength electromagnetic waves on a parallel plate photovoltaic device cause development of stresses and oscillations and results in propagation of following composite waves [6]: 1) transmission of electricity due to connected external load; 2) the heat waves are propagated at longer wavelength due to exchange of dissipated energy with medium of contact; 3) elastic waves are propagated in photovoltaic modules and polystyrene filled plywood board due to thermal stress development with propagation of heat waves; 4) fluid surface waves are propagated due to combined stress development with heat waves, elastic waves and applied external waves; 5) applied external waves are propagated due fan induced pressure and climate-particle oscillations of wind. The sound waves can propagate with combination of fluid surface waves and applied external waves such as in an organ pipe. The sound waves are also acting due to air-borne sound propagation from the surrounding environment. The propagation of acoustic waves is caused due to various stresses and oscillations of a static particle body. The stresses developed due to various forces acting on a cavity wall are classified as: 1) fundamental stresses; 2) internal stresses; and 3) external stresses. The fundamental stresses are developed due to presence of gravitational and electromagnetic forces. The internal stresses are developed under the influence of fundamental stresses and are defined by properties of a static particle body. The external stresses are developed under an external source of energy such as fan pressure used for active air ventilation through the cavity wall. The oscillations of a cavity wall are assumed to be generated because of stress development with cyclic load of day/night, heating/cooling and compression/expansion applied on a cavity wall. The climate-particle oscillations of wind are also assumed to be acting on a cavity wall. 
The acoustic waves of cavity wall are propagated due to stresses and oscillations of a cavity wall. The fluctuating forces are generated due to superposition of composite acoustic waves. The analysis has been presented for series and parallel cases of L-C-R circuit resonance. The cases of resonance can be visualised with the aid of presented experimental and modelling results. Inductance is mass for the mechanical system for propagation of elastic waves. Capacitance is heat storage capacities of photovoltaic modules, air and polystyrene filled plywood board. Polystyrene filled plywood board is vulnerable to fire as soon as heat waves propagated with frequency matching with its latent heat of vaporisation is reached. Resistance is developed due to thermal and fluid resistance in energy storage elements of photovoltaic modules, air and polystyrene filled plywood board. The series case of L-C-R resonance occurs with propagation of elastic waves of mechanical system of photovoltaic cavity wall. The parallel case of L-C-R resonance occurs with heat waves $(C-R)$ and fluid surface waves $(C-R)$ in conjuncttion with inductance (L) due to mass of photovoltaic cavity wall and resistance (R) due to temperatures of ground surface and ambient air.

\section{Conclusion}

A theory of acoustics in solar energy supporting the principle of source and sink of solar energy is presented. The speed of doing work on earth planet is directly proportional to gravitational forces (including wind and tide pressure). The gravitational forces (including wind and tide pressure) are function of solar intensity. The measurements and modelling results are presented for a parallel plate photovoltaic device connected to a potentiometer. A phenomenon of photovoltaic amplification has been formulated similar to resonance conditions applicable to the theory of sound. The resonance conditions are presented for series and parallel cases of L-C-R con- nected elements. The maximum energy transfer can occur during resonance with transmission of light, sound, noise, heat, electricity, fluid and fire from a parallel plate photovoltaic device connected to a potentiometer. The expressions for bandwidths and quality factors during occurrence of resonance at maximum energy transfer are presented. Some of the aftermaths of turning off solar energy are: 1) cooling of Earth planet; 2) gradual reducetions of gravity, wind pressure and tide pressure; 3) trapping of hydrogen fuel from ice and breaking of common salt because of split in chemical bonds; 4) transformation of an exponential function into a linear function; 5) noise behaviour of public (chaos, darkness, accidents due to zero jet lag, echoes, distorted vision of three-dimensional objects, sexual and erectile dysfunction and abnormal births of infants during post pregnancy).

\section{REFERENCES}

[1] H. Dehra, "Solar Energy Absorbers," In: R. Manyala, Ed., Solar Collectors and Panels, Theory and Applications, 2010, pp. 111-134.

[2] H. Dehra, "The Noise Scales and Their Units," Canadian Acoustics, Vol. 36, No. 3, 2008, pp. 78-79.

[3] J. W. S. Rayleigh, "The Theory of Sound," Vol. 1, Dover Publications, New York, 1894.

[4] H. Dehra, "A Numerical and Experimental Study for Generation of Electric and Thermal Power with Photovoltaic Modules Embedded in Building Façade," Submitted/Unpublished Ph.D. thesis, Department of Building, Civil and Environmental Engineering, Concordia University, Montréal, 2004.

[5] V. Del Toro, "Electrical Engineering Fundamentals," Prentice-Hall, New Delhi.

[6] H. Dehra, "A Unified Theory for Stresses and Oscillations," Canadian Acoustics, Vol. 35, No. 3, 2007, pp. 132-133. 